\title{
V. SCHLUSSBEMERKUNGEN
}

Als Robespierre auf dem Höhepunkt seiner Machtentfaltung zum Jahreswechsel 1793/94 die Revolution als "guerre de la liberté contre ses ennemis" bestimmte, brachte er das Thema der vorliegenden Arbeit auf den Punkt: Sie behandelt vor allem den Prozeß einer wachsenden diskursiven Identifikation der Revolution mit sihrem، Krieg und damit zugleich die kriegerische Prägung der Revolution im und durch den revolutionären Diskurs. Im folgenden sollen die konkreten Ergebnisse der Untersuchungen über Krieg, Militär und bürgerliche Gesellschaft im politischen Diskurs der Französischen Revolution zwischen 1789 und 1799 noch einmal in knapper Zusammenfassung präsentiert werden, bevor abschließend die Frage nach der weiterwirkenden Bedeutung der revolutionären Identifikation mit dem Krieg für die Ausbildung des modernen Militarismus diskutiert wird.

\section{Die Identifikation von Krieg und Revolution}

Ihrem Selbstverständnis nach begriff sich die Französische Revolution ursprünglich als eine ebenso kriegsgegnerische wie antimilitaristische Kraft. Dennoch fuihrte die sie von Anfang an prägende Auseinandersetzung mit den gegenrevolutionären Kräften des Ancien Régime nicht nur innerhalb weniger Jahre in den Krieg, sondern die Revolution wurde diskursiv auch so eng mit dem Krieg verbunden, daß beides, die Revolution und ihr Krieg, nicht mehr voneinander trennbar zu sein, sich vielmehr gegenseitig zu bedingen schienen. Dieser diskursive Identifikationsprozeß, der zugleich den Verlauf der Revolution insgesamt in vieler Hinsicht geprägt hat, vollzog sich auf drei strukturell unterscheidbaren Ebenen: Der Ausbildung einer spezifisch revolutionären Konzeption des Krieges (1), der Ausbildung einer spezifisch kriegerischen Konzeption der Revolution (2) und der Neukonzipierung des Verhältnisses zwischen der revolutionären Gesellschaft und dem Militär (3).

1. Der Weg der Revolution in den Krieg war geprägt von einem defensiven Bedrohungsgefühl. Ihre Vertreter rechneten damit, daß die europäischen Mächte in Frankreich intervenieren könnten, und sie wurden in dieser Befürchtung durch politische Interventionsversuche, Rüstungsmaßnahmen und machtpolitische Drohgebärden auch immer wieder bestätigt. Demgegenüber beschworen die Revolutionäre mit zunehmendem Nachdruck ihre Bereitschaft, das Vaterland der Revolution gegen jeden Angriff zu verteidigen, und sie entwarfen zugleich die Vorstellung, daß die revolutionäre Nation nicht nur im Frieden, sondern auch im Krieg den Mächten der alten Ordnung weit überlegen sei. Den Versuchen insbesondere der österreichischen Politik, gestützt auf 
militärische Drohungen direkten Einfluß auf die französische Innenpolitik zu nehmen, versuchten die Revolutionäre so durch die umgekehrte Drohung mit dem Krieg entgegenzutreten. Zielte diese Drohung lange darauf ab, die auswertigen Einmischungsversuche und Kriegsdrohungen zurückzuweisen, so gewann sie mit der Zeit eine Eigendynamik, die schließlich die fortgesetzten Rüstungsmaßnahmen und Interventionsversuche, durchaus nicht ohne Berechtigung, als Kriegserklärung deuten ließ und damit die weiterhin als defensiven Akt begriffene französische Kriegserklärung möglich machte.

Das revolutionäre Bedrohungsgefühl verband zugleich, ausgehend von der teilweise bewaffneten Emigration und den verwandtschaftlichen Bindungen zwischen den Habsburgern und den Bourbonen, die äußere und die innere Revolutionsfeindschaft $\mathrm{zu}$ einem Gesamtkomplex, wobei insbesondere dem Fluchtversuch des Königs im Juni 1791, interpretiert als Auftakt einer kriegerischen Intervention, eine katalysatorische Bedeutung zukam. Da Diplomatie und Kriegsführung in der neugeschaffenen konstitutionellen Monarchie Prärogativen der Krone geblieben waren, führten die wachsenden außenpolitischen Spannungen im Herbst 1791 zugleich dazu, daß die Politik der Staatsführung mit innerer Logik in den Mittelpunkt des revolutionären Diskurses rückte. Vor diesem Hintergrund war der Versuch, den der Revolutionsfeindschaft verdächtigen König zu einem eindeutigen Bekenntnis zur neuen Ordnung zu bewegen, aufs engste mit der Forderung verbunden, er möge gegenüber den äuBeren Einmischungsversuchen und Drohungen eine selbstbewußte, schließlich auch den Krieg einkalkulierende Politik verfolgen, und umgekehrt.

Die bereits auf Jean Jaurès zurückgehende Deutung des Krieges als Folge einer wesentlich innenpolitisch motivierten, gegen die fortdauernde Macht der Monarchie gerichteten Strategie des republikanischen Flügels der revolutionären Bewegung ist nach den Ergebnissen der hier vorgelegten politischen Diskursanalyse letztlich nicht haltbar. Zwar wurde die Forderung nach einer festen Außenpolitik, schließlich nach dem Krieg durchaus als Prüfstein für die Loyalität des Königs interpretiert, doch basierte diese Verbindung keineswegs auf einer Politik, die einseitig unter einem "Primat der Innenpolitik" gestanden hätte. Thr lag vielmehr die revolutionäre Vorstellung von einer Auflösung der Grenzen zwischen Innen- und Außenpolitik zugrunde, nach der die eigentlichen politischen Konfliktlinien nicht mehr zwischen Staaten, sondern zwischen den Völkern auf der einen, ihren despotischen Herrschern auf der anderen Seite verliefen.

Der heraufziehende Krieg wurde so als ein grenzüberschreitender, revolutionärer europäischer Bürgerkrieg konzipiert, in dem es nicht um Staaten, sondern um Prinzipien gehe. Hierin lag sowohl die Grundlage für die erstmals zum Jahreswechsel 1791/92 hervortretende revolutionäre Kriegsbegeisterung als auch für die nun einsetzende Ideologisierung des Krieges als einen Kreuzzug des revolutionären Frankreich zur Befreiung der Völker Europas. Auch 
wenn das durchaus ernst gemeint war und die französische Gesellschaft kriegerisch $\mathrm{zu}$ motivieren und $\mathrm{zu}$ mobilisieren vermochte, war in dieses Kriegsbild doch ein nationalistischer Kern eingewoben, der besonders im Zeichen des Sieges und in den damit notwendig werdenden Diskussionen über den Umgang mit eroberten Territorien und sbefreiten ` Völkern immer deutlicher hervortrat. Es ging nun doch vor allem um das Eigeninteresse der französischen Nation, um sichere Grenzen, Kontributionen und Herrschaftsansprïche, ideologisch gerechtfertigt mit ihrem spezifisch revolutionären, die $\mathrm{Zu}$ kunft der Menschheit verkörpernden Charakter. Die Chance zu einer interessegeleiteten Beendigung des Krieges im Zeichen französischer Siege ist darin allerdings nicht notwendig zu sehen. Denn zugleich trat hier eine revolutionäre ideologische Eigendynamik zutage, die den Krieg als welthistorischen Entscheidungskampf zwischen fundamental gegensätzlichen Prinzipien erscheinen ließ, in dem es keinen Verständigungsfrieden geben könne und der die Revolution so zu einem "guerre à outrance» gegen alle ihre Feinde verpflichte. Vor diesem Hintergrund spricht wenig für die oft geäußerte Ansicht, daß die Kriegspolitik des Wohlfahrtsausschusses unter Führung Robespierres eine rasche Beendigung des Krieges angestrebt oder gar, wenn sie fortgesetzt worden wäre, möglich gemacht hätte.

Auch nach den großen militärischen Siegen und der terroristischen Hybris im Frühsommer 1794 blieb die Revolution an einen nunmehr allerdings ungeliebten Krieg gebunden. Da die labile, allseits bedroht erscheinende nachrevolutionäre Ordnung nur durch einen glorreichen, die Opfer des Krieges rechtfertigenden Sieg stabilisiert werden zu können schien, gewann der Krieg nun vor allem einen innenpolitisch motivierten, die Stabilisierung der republikanischen Ordnung bezweckenden Charakter. Ein gleichberechtigter Verständigungsfrieden erschien nach dieser Perspektive dagegen als konterrevolutionäres, auf die innere Destabilisierung abzielendes Projekt der monarchistischen Opposition, darauf abzielende Initiativen wurden dementsprechend kategorisch ausgeschlossen und dem Krieg so angesichts der militärischen Stärke Englands und Österreichs weiterhin Dauer verliehen.

2. Die im Konzept des revolutionären europäischen Bürgerkrieges angelegte Auflösung der Grenzen zwischen Innen- und Außenpolitik führte nicht nur dazu, daß die Revolution dem Krieg ein spezifisches Gepräge gab. Umgekehrt gewann auch die Revolution selbst im revolutionären Diskurs wie in der revolutionären Praxis einen vom Krieg geprägten, kriegerischen Charakter. Insbesondere unter dem Eindruck der akuten militärischen Bedrohung im Sommer 1792 und 1793 rückte der Kampf gegen den mit dem feindlichen Ausland verbündeten »ennemi de l'intérieur« in das Zentrum nicht nur der französischen Innenpolitik, sondern auch des revolutionären Selbstverständnisses. Eine besondere Schubkraft gewann diese Entwicklung dadurch, daß kriegerische und revolutionäre Massenmobilisierung, wie besonders in der begriffli- 
chen Doppeldeutigkeit der levée en masse hervortrat, diskursiv aufs engste miteinander verwoben wurden. Die Revolution stand vor allem in ihrer beschleunigten Radikalisierungsphase 1792 bis 1794 nicht nur im Zeichen des Krieges, sie wurde zunehmend auch im Innern als integraler Bestandteil eines umfassenderen, revolutionären Bürgerkrieges konzipiert und nahm so zunehmend selbst einen kriegerischen Charakter an.

Geschichtsmächtig wurde dieser Prozeß zuerst mit dem Sturz der Monarchie im August 1792. Nachdem bereits der Weg in den Krieg von einer wachsenden Konfrontation zwischen den revolutionären Kräften und dem der Revolutionsfeindschaft und der Zusammenarbeit mit dem Ausland verdächtigen Hof angetrieben worden war, rückte seit Kriegsbeginn die Politik der monarchischen Staatsführung eindeutig in den Mittelpunkt des Interesses der revolutionären Öffentlichkeit. Der revolutionäre Kriegsdiskurs zeichnete sich trotz aller frühzeitig hervortretenden inneren Gegensätzlichkeiten doch durch eine grundlegende strukturelle Gemeinsamkeit aus: Der Ausgang des Krieges schien nicht auf den Schlachtfeldern ausgemacht zu werden, sondern im Innern Frankreichs. Da die Revolution im Verständnis ihrer Protagonisten den Mächten des Ancien Régime auch in den Möglichkeiten der Kriegsfuihrung grundsätzlich überlegen war, wurden die militärischen Rückschläge und Niederlagen im Frühjahr und Sommer 1792 auf die mit dem feindlichen Ausland gegen die Revolution konspirierende Politik des Hofes und der von ihm eingesetzten Militärführung zurückgeführt. Wollte man den Krieg doch noch gewinnen, war es nach dieser Logik notwendig, die offensichtlich unvollständige Revolution weiter voranzutreiben, die militärischen und staatlichen Eliten unter Druck zu setzen und sie schließlich, wenn dies nicht hinreichte, zu stürzen. Eine konkrete machtpolitische Voraussetzung für den Sturm auf die Tuilerien wurde dabei durch eine Form der kriegerischen Massenmobilisierung geschaffen, die wie selbstverständlich einen revolutionären Charakter gewann. Die fédérés aus den französischen Departements, die eigentlich nach Paris kamen, um die Hauptstadt vor der drohenden Invasion durch die Armeen des Herzogs von Braunschweig, ggf. auch gegen politisierende französische Militärführer zu schützen, richteten ihre Kampfbereitschaft statt dessen gegen den mit dem Hof identifizierten sinneren Feind und wurden zur treibenden und wohl auch entscheidenden Kraft in den revolutionären Kämpfen, die im August 1792 zum Sturz der Monarchie führten.

Die Folgezeit war geprägt von dem berühmten Konflikt zwischen Montagne und Gironde, der im Licht der hier vorgelegten politischen Diskursanalyse wesentlich als ein Kriegskonflikt gedeutet werden muß. Im Kern der Auseinandersetzungen stand die Frage, ob der Krieg eher durch die innere Konsolidierung, oder aber durch die weitere Revolutionierung der Republik erfolgreich geführt werden könne. Seine tödliche Zuspitzung gewann dieser Bruderkrieg im revolutionären Lager aber dadurch, daß beide Seiten die 
Gegenposition nicht nur als politischen Fehler werteten, sondern sie wie selbstverständlich auch als Politik im Interesse der äußeren Gegenrevolution, ja als konspirative Tätigkeit bezahlter feindlicher Agenten denunzierten. Wie gezeigt werden konnte, basierten diese Denunziationen auf Denkfiguren, die bereits im Zusammenhang der Diskussionen über den heraufziehenden Krieg zum Jahreswechsel 1791/92 vorgeprägt worden waren, die im Zeichen der desaströsen militärischen Entwicklungen des Frühsommers 1792 eine verschärfte Ausprägung erfahren hatten und die nun seit dem Herbst 1792 in das Zentrum der Auseinandersetzungen zwischen gemäßigten und radikalen Revolutionären rückten. Die Entscheidung zugunsten der Montagne wird schließlich nur im Zusammenhang der erneuten Zuspitzung der militärischen Lage und mit dem Beginn des Bürgerkrieges im Innern Frankreichs im Frühjahr 1793 verständlich, deren Koinzidenz der Vorstellung von einem revolutionären Bürgerkrieg an zwei Fronten weiter Nahrung gab und der Option für die weitere revolutionäre Radikalisierung zum Sieg verhalf. Mit der Hinrichtung der Girondisten als Agenten des feindlichen Auslandes wurde im Herbst 1793 dann eine Grenzüberschreitung vollzogen, die dem Terror endgültig auch Einla $\beta$ in die innerrevolutionären Auseinandersetzungen verschaffte.

Die Entstehung des Terrorregimes vollzog sich im Spätsommer 1793, und sie wurde wesentlich angetrieben von einem erneuten Schub kriegerisch-revolutionärer Massenmobilisierung. Nach den Ergebnissen dieser Untersuchung kann nicht die Rede davon sein, daß die Ausrufung der levée en masse am 23. August und die offizielle Inaugurierung des Terrors am 5. September 1793 nur in einer zufälligen zeitlichen Nähe und nicht in einem inneren $\mathrm{Zu}$ sammenhang miteinander gestanden hätten. Vielmehr zeichnete sich die Bewegung für eine allgemeine Massenmobilisierung zur Verteidigung der vom äußeren Krieg und vom inneren Bürgerkrieg bedrohten Republik schon lange vorher dadurch aus, daß sie den Kampf gegen den sinneren` und säußeren Feind diskursiv miteinander verband, Krieg und Revolution ineinander aufgehen ließ und der revolutionären Wendung gegen den wennemi de l'intérieur' in Analogie zur revolutionären Kriegsführung gegen den äußeren Feind einen ausgeprägt terroristischen Charakter verlieh. Die levée en masse sollte im Verständnis der Pariser Volksbewegung nicht nur eine kriegerische Massenbewegung gegen den räußeren` Feind einleiten, sondern zugleich auch eine selbstorganisierte, revolutionäre Massenerhebung gegen alle Feinde der Revolution im Innern Frankreichs. Auch der Kampf im Innem sollte nun durch armées révolutionnaires geführt werden, die inneren Gegner der Revolution sollten nicht nur in Angst und Schrecken versetzt, sondern schließlich ebenso wie die feindlichen Armeen vernichtet werden. Der jakobinischen Revolutionsführung paßte eine unkontrollierbare revolutionäre Massenerhebung >von untenc allerdings nicht in ihr politisches Konzept, ihr ging es vor allem um die organisierte Mobilisierung aller Kräfte der Nation für 
die militärische Verteidigung der Revolution, und das Dekret über die levée en masse spiegelt ihren Erfolg wieder. Die nachgeschobene Forderung der revolutionären Volksbewegung, nun auch den ursprünglich im Konzept der levée en masse bereits angelegten Terror explizit auf die Tagesordnung der Revolution zu setzen, ist so als Versuch zu werten, das scheiternde Konzept der selbstorganisierten revolutionären Massenerhebung doch noch zu retten.

Das Ergebnis dieser Auseinandersetzungen über die Konzipierung des nach außen und innen gerichteten Verteidigungskampfes der Revolution war die Ausbildung der revolutionären Kriegsdiktatur der großen Ausschüsse. Sie konnte dank der revolutionären Massenmobilisierung auf enorme menschliche und materielle Ressourcen zurückgreifen und schließlich an allen Fronten von Krieg und Bürgerkrieg militärisch triumphieren. Zugleich aber war ihr konzeptionell ein terroristischer Charakter eigen, der sich im Kampf gegen den zum »étranger de l'intérieur" erweiterten sinneren Feind، zunehmend jeder instrumentellen Eingrenzung entzog, eine eigene Qualität gewann und eine sich radikalisierende Dynamik ausbildete. Die terroristische Hybris der Revolution ist demnach nicht, wie die $\mathrm{z}$. Zt. dominierende revisionistische Revolutionsdeutung meint, primär als Folge eines übersteigerten Tugendbegriffs zu verstehen, der einer widerstrebenden Gesellschaft aufgezwungen werden sollte. Ebensowenig aber war sie, wie die apologetische Einordnung lautet, eine notwendige Folge der prekären Lage einer Revolution, die nur mit Hilfe des Terrors ihren Kampf gegen die äußere und innere Konterrevolution hätte gewinnen können. Der Terror entsprang nicht dem Krieg an sich, sondern der Konzeption des revolutionären Bürgerkrieges, die die äußeren und inneren Gegner der Revolution zu einer gemeinsamen Bedrohung verband, gegen die ein so kriegerischer wie revolutionärer Kampf an zwei unauflöslich miteinander verbundenen Fronten geführt werden müsse.

Die Eigendynamik dieser forcierten Identifikation von Krieg und Revolution führte schließlich dazu, alle inneren Probleme, Abweichungen und Konflikte ihres Eigenwertes zu entkleiden, sie als Bestandteile im allgemeineren $\mathrm{Zu}$ sammenhang des revolutionären, an zwei Fronten gefuihrten Bürgerkrieges zu interpretieren und ihre deutlicheren Ausprägungen auf konspirative Tätigkeiten des feindlichen Auslandes zurückzuführen, die es mit terroristischer $\mathrm{Ge}$ walt zu bekämpfen gelte. Nur in diesem Zusammenhang wird es verständlich, wieso die Revolutionsführung Anfang 1794 sowohl den gemäßigten als auch den radikalen Flügel der revolutionären Bewegung als Bedrohung der Revolution interpretieren und ihre Führer als feindliche Agenten aufs Schafott schicken konnte. Wenn die militärischen Siege der Revolutionsarmeen im Frühjahr 1794 dann nicht zum Abklingen, sondern zu einer weiteren Ausweitung des revolutionären Terrors führten, so kann dies keineswegs als Beleg dafür gelten, daß sich der Terror inzwischen grundsätzlich vom Krieg abgelöst hätte. Er blieb vielmehr einem radikalisierten Verständnis des revolutionären 
Bürgerkrieges verhaftet, das gerade angesichts der militärischen Überlegenheit davon ausging, der Feind werde nun um so mehr auf konspirative Aktivitäten innerhalb Frankreichs zurückgreifen. Nach dieser Logik lauerten die Feinde der Revolution nun überall, jede Auffälligkeit geriet unter Verdacht und die vermeintliche Allgegenwart des Feindes führte umgekehrt zur Allgegenwart des Terrors. Die schließlich als Vernichtungskrieg gegen alle ihre Feinde begriffene Revolution konnte kein Ende mehr finden, bevor nicht die Mehrheit ihrer Protagonisten selbst sich so sehr bedroht fühlte, daß sie der terroristischen Hybris ein Ende setzte. Dies wurde aber erst möglich, als die französischen Armeen an allen Front siegreich waren und so die diskursive Verbindung von äußerer und innerer Bedrohung, von Krieg und Terror ihre eigentliche Grundlage verloren hatte.

Die diskursive Bindung der Revolution an ihren Krieg fand damit indes kein Ende. So wie ein glorreicher Sieg die in der Revolution geschaffene neue Ordnung stabilisieren sollte, so diente der damit verewigte Krieg nun zugleich der inneren Herrschaftslegitimation. Die nun herrschende, gemäßigt- revolutionäre Elite sah sich einer prekären Minderheitssituation gegenüber, bedroht sowohl von revolutionären wie von konterrevolutionären Kräften und Aufständen. Ihre wechselnden Schläge gegen die Opposition von rechts und von links wurden weiterhin im Rahmen des Konzeptes eines grenzüberschreitenden revolutionären Bürgerkrieges als Kampf gegen den mit dem feindlichen Ausland verbündeten sinneren Feind legitimiert, doch trat dabei der instrumentelle, auf die Ausschaltung politischer Gegner zielende Charakter immer deutlicher zutage. Paradoxerweise trug diese den Krieg zur Herrschaftssicherung instrumentalisierende Politik aber nicht nur zur Verewigung des Krieges bei, sondern auch zur Untergrabung der inneren Ordnung, die sie doch stabilisieren sollte. Denn gerade auf der Basis des zunehmend die Verfassung mißachtenden Kampfes gegen den sinneren Feind, von rechts und links wurde eine innere Konsolidierung der Republik, die doch die Integration unterschiedlicher politischer Tendenzen in die neue Verfassungsordnung zur Voraussetzung gehabt hätte, immer unwahrscheinlicher. Zugleich aber unterstützte die militärisch abgesicherte Politik des Staatsstreiches den Aufstieg eines neuen Machtfaktors, der die republikanische Ordnung schließlich umstürzen sollte.

3. Tiefgehend geprägt wurde die Französische Revolution schließlich von der diskursiven Neukonzipierung des Verhältnisses zwischen Zivilgesellschaft und Militär. Nicht mehr die Trennung zwischen ihren disparaten Bereichen stand im Mittelpunkt des revolutionären Diskurses, sondern umgekehrt ihre Bezüge und Wirkungsverhältnisse. Das Militär wurde von den Revolutionären zu Anfang als die eigentliche Stütze der alten Ordnung und damit als ihr ernstzunehmenster Gegner betrachtet. Die - durchaus nicht unberechtigte Angst vor einem konterrevolutionären Einsatz der bewaffneten Macht und die 
Konfrontation mit dem Militär rückte so in das Zentrum des revolutionären Diskurses und wurde darüber zu einer Triebkraft der revolutionären Bewegung, wie sie vor allem beim Sturm auf die Bastille, beim Marsch der Pariser Bevölkerung nach Versailles und in der Föderationsbewegung 1790 geschichtsmächtig Ausdruck fand.

Angesichts des Gefühls der Bedrohung durch das königliche Militär sah sich die revolutionäre Bewegung zuerst zur spontanen Bewaffnung, dann zum Aufbau einer selbständigen bewaffneten Gegenmacht veranlaßt, die in der Nationalgarde ihre organisatorische Form fand. Die diskursive Durchdringung des revolutionären Bürgers, des bewaffneten Kämpfers und des die neue Macht symbolisierenden Nationalgardisten trug entscheidend dazu bei, das Bild des revolutionären Bürgers zu prägen und das aktive Bürgerrecht auf waffenfähige Männer zu begrenzen. Die neue Konzeption des citoyen soldat sollte vor allem aber dazu dienen, die alte königliche Armee zu zivilisieren und eine neue, den Vorrang der zivilen vor der militärischen Ordnung sicherstellenden Organisation der bewaffneten Macht zu begründen. Da dies der verfassungsgebenden Nationalversammlung nach dem Verständnis vieler Revolutionäre sowohl in der traditionellen Armee als auch in der neuen Nationalgarde unter ihrem so charismatischen wie undurchsichtigen General La Fayette nur teilweise gelang, blieb das revolutionäre Mißtrauen gegen die bewaffnete Macht wach und rückte mit der wachsenden außenpolitischen Konfrontation und dem beginnenden Krieg erneut in das Zentrum der politischen Auseinandersetzungen. Während die revolutionär engagierten Bürgersoldaten nun als Garanten des Sieges galten, wurden militärische Rückschläge einer der Monarchie verpflichteten Generalität angelastet, die sowohl der Zusammenarbeit mit dem militärischen Gegner als auch selbständiger konterrevolutionärer Ambitionen verdächtig schien. Nicht zuletzt gegen diese Bedrohung richtete sich der Sturm auf die Tuilerien. Doch im Diskurs der radikalen Kräfte konnte sich der Verdacht gegen die Militärführung schließlich auch gegen alle diejenigen Kräfte im revolutionären Lager richten, die mit ihrem Votum für den Krieg vermeintlich den politisierenden Generälen zugearbeitet oder ihnen gar Vertrauen entgegengebracht hatten.

Im Zeichen der Forderung nach einer revolutionären, die gesamte Gesellschaft erfassenden levée en masse zum Entscheidungskampf für die Revolution kam es $1793 \mathrm{zu}$ einer kurzen Phase der allgemeinen diskursiven Durchdringung von Militär und Zivilgesellschaft, die jedoch bald wieder abgelöst wurde von neuen Formen der Konfrontation. Nicht mehr um die zivilisierende Eingrenzung und Umgestaltung des monarchischen Militärs als Stütze der alten Ordnung durch die revolutionären Bürger ging es nun, sondern umgekehrt um die Prägung und Beherrschung der zivilen Gesellschaft durch das revolutionäre, zunehmend in Gesellschaft und Politik intervenierende Militär. Hatten die Bürgersoldaten bislang als militärische Speerspitze 
einer primär zivilen revolutionären Bewegung gegolten, so erschien das zunehmend verselbständigte Militär im politischen Diskurs der zweiten Hälfte der 1790er Jahre als Garant, ja als Inkarnation der Revolution. Vor allem in den Militärzeitschriften wurde, gestützt auf durchaus vorhandene soziale Gegensätze, nun ein Bild gezeichnet, in dem das Militär zum Hort revolutionärer Tugenden stilisiert und mit einer vermeintlich verkommenen Zivilgesellschaft konfrontiert wurde, die sich von den revolutionären Idealen abgewandt habe, nur noch ihre Partikularinteressen verfolge und nun der Erneuerung durch das revolutionäre Militär und seiner Tugenden bedürfe. So entstand der Anspruch der Soldaten und vor allem ihrer Generäle, Politik und Gesellschaft nach ihren eigenen, höherwertigen Prinzipien neu zu ordnen, und General Bonaparte wurde, gestützt auf die triumphalen Erfolge im Italienfeldzug, sein führender Vertreter. Der vom Militär unter Anführung von Bonaparte mit großem Nachdruck geforderte, ja geradezu erzwungene und schließlich auch mit ziviler Einwilligung durchgeführte Staatsstreich vom 18. Fructidor des Jahres V kann schließlich als Generalprobe für den Militärputsch begriffen werden, mit dem der General gut zwei Jahre später, am 18./19. Brumaire des Jahres VII, die republikanische Ordnung umstürzte und selbst an die Macht gelangte.

Der Machtergreifung Bonapartes ging eine erneute Zuspitzung der militärischen Situation der Republik voraus, die dazu führte, daß die zentralen Topoi des revolutionären Kriegsdiskurses noch einmal aktiviert wurden: die ideologische Legitimation des revolutionären Bürgerkrieges gegen die alte Ordnung, der revolutionäre Kampf gegen den Feind im Innern und die so kriegerische wie revolutionäre Mobilisierung der Bevölkerung. Als Inkarnation dieser revolutionären Traditionen aber galt nun vor allem das Militär und besonders sein Héros Bonaparte. Der spezifische Diskurs des militärischen Staatsstreiches stützte sich zum einen auf die charismatische, die kriegerische Tradition der Revolution verkörpernde Figur des Generals, zum anderen verband er die diskursiven Topoi des kriegsbedingten Kämpfen gegen den sinneren Feind und der Konfrontation zwischen dem glorreichen revolutionären Militär auf der einen, den verkommenen, die Revolution verratenden zivilen Gewalten auf der anderen Seite, zu einer ebenso mitreißenden wie explosiven Mischung. Alle alternativen politischen Projekte von der Bewahrung des status quo über die Verfassungsrevision bis zur neojakobinisch-revolutionären Radikalisierung blieben demgegenüber ohne vergleichbare Inspirationskraft. Nachdem die Neojakobiner lange gemeinsam mit der parti des généraux die etablierte direktoriale Ordnung bekämpft hatten, löste sich Bonaparte schließlich auch von ihren revolutionären Projekten und entmachtete, ebenso gestützt auf den militärisch-revolutionären Diskurs wie auf die Macht der Bajonette, die diskreditierten zivilen Gewalten und etablierte trotz aller zivilen Verkleidungen in den machtpolitischen Grundzügen de facto eine Militärdiktatur. 


\section{Die Erfindung des modernen Militarismus}

Für Jean Jaurès, der nicht nur ein bedeutender Historiker und Politiker war, sondern auch ein kluger Analytiker von Krieg und Militär, markierte der Hébertismus wla première apparition du militarisme dans la Révolution française«. Denn der Hébertismus sei, so die Begründung, "militariste par son origine même; c'est dans le bureau de la guerre qu'il est né et qu'il a grandi. Il a le sabre traînant et la moustache provocatrice. Il est militariste par le goût de la brutalité et de la parade. Il se plaît aux expéditions de l'armée révolutionnaire, aux gestes terrifiants qui ressemblent aux allures d'une armée en pays conquis. Et ces chefs populaires (...) se couvrent de dorures pour marcher derrière la guillotine, comme les prêtres se revêtent d'étoiles splendides pour aller sous le dais. Il est militariste par l'horreur et la crainte du pouvoir civil. Casser la Convention lui serait un jeu, et il installerait au pouvoir, le lendemain, tout un état-mayor à panaches. (...) L'hébertisme était militariste par sa complaisance à une politique prolongée d'exécutions militaires. Son idéal révolutionnaire, c'était, le lendemain d'un assaut, les sauvages représailles de guerre sur les villes vaincues «'.

Diese von der Abneigung des humanitären Sozialisten gegen jede Form von Militarismus getragene Charakterisierung weist in eindringlicher Form darauf hin, daß im Zusammenhang des revolutionären Krieges gegen den sinneren Feind in der Radikalisierungsphase der Revolution mit den paramilitärischen armées révolutionnaires und ihren politisierenden Führern zweifellos Formen von Militarismus entstanden sind. Zugleich aber bleibt das Urteil von Jaurès, da es sich hier nicht um eine allgemeinere Einordnung des Phänomens in die Revolutionsgeschichte bemüht, letztlich doch in mancher Hinsicht an der Oberfläche stehen. Einmal abgesehen davon, daß die intensiven Untersuchungen von Richard Cobb trotz der kriegerischen Attitüde auf einen in vieler Hinsicht eher zivil-revolutionären als spezifisch militärischen Charakter der armées révolutionnaires hindeuten, ist in ihren zweifellos dennoch erkennbaren militaristischen Erscheinungsformen vor allem kein wirklich prägendes Element der Radikalisierungsphase der Revolution in den Jahren 1792 bis $1794 \mathrm{zu}$ erkennen. Vielmehr handelt es sich dabei um ein eher untergeordnetes Phänomen im Rahmen der das politische Geschehen bestimmenden Konzeption eines revolutionären Bürgerkrieges, dessen wesentliches Charakteristikum nicht in einem Dominanzanspruch des Militärs oder des Militärischen $\mathrm{zu}$ sehen ist, sondern in der Umformung des kämpferischen Selbstverständnisses der Revolution zu einem kriegerischen Selbstverständnis. Diese Umformung basierte auf der wechselseitigen Identifikation von krie- 
gerischer Mobilmachung und revolutionärem Kampf, von Revolution und Krieg, und sie brachte deutliche Tendenzen einer inneren Militarisierung der Revolution mit sich. Der eigentliche revolutionäre Militarismus mit seinem dezidierten Anspruch auf den Vorrang des nun die Revolution verkörpernden Militärs vor der zivilen, als dekadent denunzierten Gesellschaft und Politik entwickelte sich jedoch erst in den darauf folgenden Jahren, und er war aufs engste mit der Verselbständigung des Militärs nach dem Sturz der Terrorherrschaft und vor allem mit dem Aufstieg General Bonapartes verbunden.

So vielfältig und unpräzise der Militarismus-Begriff sich oft darstellt, grundlegend erscheint die Verselbständigung des Militärs und des Militärischen gegenüber den Formen, Zielen und Inhalten ziviler Politik und gesellschaftlicher Ordnung ${ }^{2}$. Als militaristisch können dabei einzelne Erscheinungsformen charakterisiert werden, doch als umfassender Systembegriff ergibt "Militarismus" nur dann einen Sinn, wenn seine in Politik, Ideologie und Gesellschaft hineinreichenden Wirkungen durch den Bezug auf das Militär selbst, auf seine Machtansprüche und seine politisch wie gesellschaftlich prägende Kraft zusammengebunden werden. Gleichwohl erscheint es sinnvoll, die Frage nach den strukturell prägenden Wirkungen der Französischen Revolution für die Entwicklung des modernen Militarismus nicht allein auf die Entwicklung des Verhältnisses zwischen Militär und ziviler Gesellschaft zu begrenzen, sondern sie mit einer weiter gefaßten, das allgemeinere Verhältnis von Revolution, Krieg und Militär einbeziehenden Perspektive zu verbinden. Denn ohne die neuartige Konzipierung des revolutionären Krieges und, eng damit verbunden, die Ausbildung der revolutionären Kriegsideologie sowie die kriegerische, sich zugleich gegen den sinneren Feind، richtende Mobilisierung der revolutionären Gesellschaft kann weder die Verselbständigung des revolutionären Militärs während der Revolution noch die weitere Entwicklung des modernen, nicht nur auf die politische, sondern auch auf die gesellschaftliche Herrschaft zielenden Militarismus angemessen erklärt werden. Auch wenn diese Topoi des revolutionären Kriegsdiskurses im zeitlichen Ablauf vor und unabhängig von dem neuartigen, revolutionär begründeten, militärischen Herrschaftsanspruch entstanden sind, haben sie sich in der weiteren historischen Entwicklung doch so eng mit der Militärherrschaft verbunden, daß sie tatsächlich als integrale Bestandteile eines allgemeiner bestimmten Begriffs des modernen, in der Französischen Revolution geprägten Militarismus zu verstehen sind.

Ursächlich ist Militarismus in der Regel mit Formen präindustrieller, feu-

2 Vgl. allg. V. R. BERGHAHN (Hg.), Militarismus, Köln 1975; DERS., Militarismus. Die Geschichte einer internationalen Debatte, Hamburg u. a. 1986; W. CONZE, M. GEYER, Militarismus, in: Geschichtliche Grundbegriffe. Historisches Lexikon zur politisch-sozialen Sprache in Deutschland, hg. v. O. BRUNNER, W. CONZE, R. KOSELLECK, 6 Bde., Stuttgart 1972ff., hier Bd. 4, S. 1-47; ferner W. JANSSEN, Krieg, in: ebd., Bd. 3, S. 567-615. 
dalstaatlicher Gewaltorganisation, oder aber alternativ mit der kriegerischen Formierung moderner industrieller Klassengesellschaften und ihrer immensen Gewaltpotentiale verbunden worden. Die extremen Ausprägungen und Exzesse des Militarismus im 20. Jahrhundert erscheinen so als Mischformen, die gerade durch die enge Durchdringung von industriegesellschaftlichen Gewaltpotentialen und feudalen Überhängen in den sozialen und politischen Machtverhältnissen, wie sie besonders für die preußisch-deutsche und die japanische Geschichte aufgezeigt worden sind, eine extreme Radikalisierung erfahren haben. Wenn hier nun die grundlegende Bedeutung der Französischen Revolution für die Ausbildung des modernen Militarismus betont wird, so soll die skizzierte Auffassung damit keineswegs grundsätzlich verworfen werden. Die Revolution selbst war anfangs ihrem inneren Wesen nach, wie hier gezeigt werden konnte, keineswegs bellizistisch oder gar militaristisch geprägt, und die moderne bürgerliche Gesellschaft hat in ihrer weiteren Entwicklung vielfältige Elemente hervorgebracht, die durchaus mit Erfolg das Militär und den Krieg einhegen konnten. Umgekehrt waren es tatsächlich oft vormoderne Überhänge, die gerade die Ausbildung solcher Begrenzungen behindert und damit der uneingeschränkten Herrschaft des Militarismus den Weg bereitet haben. Trotzdem aber waren die inhaltlichen Grundformen des modernen, die Potentiale der bürgerlichen Gesellschaft in seinem Sinne entfaltenden Militarismus, das zeigen die Ergebnisse dieser Arbeit ebenfalls deutlich, schon in der Französischen Revolution und ihrer Auseinandersetzung mit den Kräften der alten Ordnung angelegt. Es liegt demnach nahe, sie nicht als Fremdkörper, sondern als Tendenzen, als Entwicklungsmöglichkeiten anzusehen, die der modernen bürgerlichen Gesellschaft grundsätzlich eingeschrieben sind.

Läßt man die schon oft betonte Bildung neuartiger Massenheere sowie die Besatzungspraxis der revolutionären Armeen beiseite und konzentriert sich auf das im politischen Diskurs wesentlich hervortretende Selbstverständnis der revolutionären Gesellschaft, so sind drei eng mit Krieg und Militär verbundene Strukturkomplexe der Französischen Revolution hervorzuheben, durch die etwa der friederizianische Militärstaat als Inkarnation des traditionellen Militarismus gerade nicht ausgezeichnet war und die die Entwicklung des modernen Militarismus nachhaltig geprägt haben: Die Entstehung einer modernen Kriegsideologie und der Entwurf des Weltanschauungskrieges (1), die Tendenz zum totalen, auch die Zivilbevölkerung aktiv einbeziehenden Krieg und, in enger Verbindung damit, der terroristische Kampf gegen den sinneren Feind (2), schließlich die Ausbildung eines eigenständigen militärischen Selbstverständnisses und eines politischen Gestaltungsanspruchs des Militärs gegenüber Staat und Gesellschaft als eigentlichem Kernelement des modernen Militarismus (3). 
1. Die Entwicklung der revolutionären Kriegsideologie war gekennzeichnet durch drei Aspekte, die in struktureller Form die moderne Kriegsideologie schlechthin vorgezeichnet haben. Vor dem Hintergrund ihres kriegsgegnerischen Selbstbildes einerseits, einer demokratisch geprägten Öffentlichkeit andererseits, konnte die Revolution ihren Krieg gegen die Mächte der alten Ordnung nur als einen Verteidigungskrieg begreifen. Wie gezeigt wurde, war die Kriegsschuld tatsächlich keineswegs einseitig verteilt. Doch aus sich selbst brachte die Revolution die für jede moderne Kriegsideologie grundlegende Stilisierung eines gerechten Verteidigungskrieges hervor, an dem sie auch noch, nun in eindeutig ideologisierter Weise, festhalten konnte, als sie sich längst einer offensiven Kriegspolitik verschrieben hatte oder gar - hier gehen wir zum zweiten Aspekt über - expansionistische Ziele zu verfolgen begann. Ihrem ideologisierten Selbstbild nach kämpfte die Revolution jedoch nicht um Machtinteressen. Der Krieg wurde vielmehr als ein Kampf zwischen antagonistischen gesellschaftlichen Werten und Prinzipien begriffen, in dem die eigene Seite, auch dies ein Kernelement moderner Kriegsideologie, nicht primär nationale Eigeninteressen $z u$ vertreten schien, sondern den gesellschaftlichen Fortschritt schlechthin und damit auch die wahren Interessen der gegnerischen Bevölkerung und die Zukunft der Menschheit. Trotzdem verband sich mit dieser Form des modernen Weltanschauungskrieges zum dritten auch die moderne, kriegerische Radikalisierungsprozesse legitimierende Vorstellung, da $B$ der Krieg für den Fortschritt der Menschheit und gegen die Mächte der Reaktion, in verallgemeinerter Form für das von der eigenen Nation repräsentierte Prinzip und gegen die Verkörperung des feindlichen Prinzips, "à outrance«, also bis zur Vernichtung des gegnerischen Prinzips und der es verkörpernden Kräfte, oder aber bis zum eigenen Untergang geführt werden müsse. Zusammengefaßt entstanden aus der Ideologie des revolutionären Bürgerkrieges unter den Bedingungen eines Krieges zwischen Nationen und Staaten die ideologischen Grundlagen des kriegerischen Nationalismus und des nationalistischen Volkskrieges.

2. Mit der Konzeption des grenzüberschreitenden revolutionären Bürgerkrieges waren weitere, nach innen zielende Tendenzen verbunden, die auch für den modernen Militarismus generell eine grundlegende Rolle spielen und insbesondere als wesentliche Voraussetzungen sozialer Militarisierung zu begreifen sind. Dies war zum einen, in enger Verbindung mit der Ideologisierung des Krieges, die neuartige Idee eines totalen, nicht nur die zunehmend konskribierten Bürgersoldaten, sondern auch die gesamte zivile Bevölkerung, die ২Heimatfront؛, prinzipiell für die Kriegsanstrengungen mobilisierende Kriegspolitik, wie sie vor allem in dem berühmten Aufruf zur levée en masse 1793 in zwar gewissermaßen naiver, politisch nur ansatzweise umgesetzter, semantisch aber doch deutlich ausgeprägter Form hervortrat. Alle privaten Belange sollten angesichts der Bedrohung der Nation hinter den Notwendig- 
keiten einer umfassenden Mobilisierung für die nationalen Kriegsanstrengungen zurückstehen, die alle waffenfähigen Männer zum Kriegsdienst an der Front, alle anderen zum uneingeschränkten Einsatz für die Belange der Kriegsführung im Landesinnern verpflichtete. Wer sich dem nicht fügen wollte, geriet in den Verdacht, die Landesverteidigung zu boykottieren und im Dienste des Feindes zu stehen. Zum zweiten handelte es sich um die moderne Formen einer Militarisierung der Politik und der Legitimation politischer Herrschaftsansprüche des Militärs generell vorzeichnende Wendung nicht nur gegen den >äußeren،, sondern zugleich auch gegen den vermeintlich mit diesem verbundenen sinneren Feind،. Erst am Ende des Revolutionsprozesses wurde dieser Topos des revolutionären Bürgerkrieges mit dem Herrschaftsanspruch des Militärs verbunden, doch fortan konnte der Kampf gegen den sinneren Feind، immer wieder als Legitimation für die Ersetzung vermeintlich dazu nicht fähiger ziviler Autoritäten durch das kampferprobte, zum inneren Bürgerkrieg bereite Militär dienen. Im Zeichen der Identifizierung von Krieg, Bürgerkrieg und Revolution fand der Kampf gegen den sinneren Feind in der Französischen Revolution schließlich eine terroristische, nicht nur auf Abschreckung, sondern auch auf Vernichtung des politischen Gegners abzielende Zuspitzung, wie sie für die Praxis moderner Militärdiktaturen und militaristischer Radikalisierungsprozesse generell als typisch gelten kann. So entstand aus der Konzeption des revolutionären Krieges der moderne, militarisierte Staatsterror, bevor die Revolution nach der terroristischen Hybris mit der Fortsetzung des Krieges als Mittel der Machterhaltung und seiner Instrumentalisierung für den innenpolitischen Kampf schließlich auch die herrschaftslegitimatorische Funktion des modernen Krieges entdeckte. In der diskursiven Identifikation von Krieg und Revolution war schließlich bereits die so typisch militaristische, viel später erst sozialdarwinistisch fundierte Vorstellung angelegt, daß der Krieg selbst eine regenerative Kraft für die zivile Gesellschaft besitze, ja ein notwendiger Faktor der gesellschaftlichen Entwicklung sein könne. Der kommende Krieg, so hatte Brissot bereits Ende 1791 mit Blick auf den inneren Zustand der Gesellschaft prognostiziert, "sera un bienfait, un bienfait national«. Und Billaud-Varenne, ehemals ein erklärter Kriegsgegner, kam gut zwei Jahre später zu dem Schluß: »La guerre, qui semblait devoir consommer notre ruine, est pourtant ce qui nous a sauvé ...«.

3. Das Verhältnis zwischen Militär und ziviler Gesellschaft, zwischen Bürgern und Soldaten, erfuhr im Zusammenhang des Krieges schließlich eine fundamentale Umkehrung, die den modernen Militarismus im engeren Sinne überhaupt erst hervorgebracht hat. Hatte die Revolution mit der Konzeption des soldat citoyen anfangs die Zivilisierung des Militärs angestrebt, dann im Zeichen des Krieges die immer umfassendere, zuerst freiwillige, dann auch zwangsweise kriegerische Mobilisierung der Bevölkerung betrieben, so verschob sich im weiteren Verlauf unter den Bedingungen des nicht endenden 
Krieges noch einmal die Perspektive. In den Vordergrund trat nun die Ausbildung eines eigenständigen militärischen Selbstverständnisses und die Beherrschung der zivilen Politik und Gesellschaft durch das Militär. Militarismus in der engen Definition von Gerhard Ritter, begriffen als Vorrang der militärischen vor der zivilen Entscheidungskompetenz $z^{3}$, bildete sich spätestens im Zusammenhang von Bonapartes Italienfeldzug aus, als der General nicht nur die militärische Strategie, sondern auch die Kriegspolitik in seine Hände nahm, selbständig Friedensverhandlungen führte, Verträge schloß und die politische Ordnung Italiens nach eigenem Gutdünken umzugestalten begann. Diese Politik verband sich im militärischen Diskurs jedoch mit weiterführenden, in neuartiger, nur vor dem Hintergrund der Revolution verständlicher Weise auf die soziale Militarisierung der zivilen Gesellschaft und die militärische Übernahme der politischen Macht abzielenden Ansprüchen.

Die im militärischen Diskurs der zweiten Hälfte der 1790er Jahre ausgebildete Konfrontation zwischen dem Militär und der zivilen Gesellschaft brachte mit dem von den Soldaten formulierten Anspruch, die wahren Werte der Revolution zu verkörpern und gegenüber einer als verkommen abqualifizierten zivilen Gesellschaft zu vertreten, zum einen den Grundansatz der modernen sozialen Militarisierung hervor: Die zivile Gesellschaft sollte zu ihrer inneren Gesundung von militärischen Eigenschaften, Tugenden und Organisationsformen durchdrungen und geprägt werden. Die Ende 1798 eingeführte Allgemeine Wehrpflicht war dafür zweifellos förderlich, doch im Zentrum dieses Projektes stand sie nicht. Zum anderen beinhaltete diese Konfrontation zunehmend auch den die moderne Militärdiktatur vorprägenden Anspruch, daß das Militär selbst in direkter Weise die politische Macht an sich reißen müsse. Nach einigen Vorläufen etablierte schließlich die auf das eigenständige Selbstbewußtsein des Militärs als Garant der revolutionären Tradition - und selbstverständlich auf die Macht der Bajonette - gestützte Absetzung der legalen Autoritäten der Republik durch General Bonaparte den modernen Militärputsch und die darin angelegte Herrschaft des Militärs.

Der moderne, mit Bonaparte siegreiche Militarismus zielte auf die vollständige militärische Beherrschung und Gestaltung der zivilen Gesellschaft. Seine raison d'être aber blieb der Krieg, der ideologisch legitimierte, die ganze Gesellschaft mobilisierende moderne Nationalkrieg. Obwohl der General sich anfangs erfolgreich um die innere und äußere Befriedung, um die Beendigung des dynamischen Beziehungsgeflechtes von Krieg und Revolution bemühte, trat der kriegerisch-militaristische Charakter seiner Herrschaft so doch schon bald wieder deutlich zutage. Als Bonaparte, um eine Denkfigur des jungen Marx aufzugreifen und zugleich abzuwandeln, den ideologisierten revolutio-

3 Vgl. G. RITTER, Staatskunst und Kriegshandwerk. Das Problem des Militarismus in Deutschland, 4 Bde., München 1954ff. 
nären Terrorismus gegen die reale bürgerliche Gesellschaft in militaristischer Form fortfuihrte, indem er die permanente Revolution durch den permanenten Krieg ersetzte, brauchte er tatsächlich nur den Schwerpunkt im komplexen Beziehungsverhältnis zwischen der Revolution und ihrem Krieg noch ein Stück weiter auf die Seite des Krieges zu verlagern.

4 Vgl. K. MaRX, F. Engels, Die heilige Familie oder Kritik der kritischen Kritik. Gegen Bruno Bauer und Konsorten (1845), in: MEW, Bd. 2, S. 3-224, hier S. 130. 